\title{
Molecular cloning of chicken aggrecan
}

\author{
Structural analyses
}

\author{
Lakshmi CHANDRASEKARAN and Marvin L. TANZER* \\ Department of BioStructure and Function, School of Dental Medicine, University of Connecticut Health Center, \\ Farmington, CT 06030, U.S.A.
}

\begin{abstract}
The large, aggregating chondroitin sulphate proteoglycan of cartilage, aggrecan, has served as a generic model of proteoglycan structure. Molecular cloning of aggrecans has further defined their amino acid sequences and domain structures. In this study, we have obtained the complete coding sequence of chicken sternal cartilage aggrecan by a combination of cDNA and genomic DNA sequencing. The composite sequence is $6117 \mathrm{bp}$ in length, encoding 1951 amino acids. Comparison of chicken aggrecan protein primary structure with rat, human and bovine aggrecans has disclosed both similarities and differences. The domains which are most highly conserved at $70-80 \%$ identity are the $N$-terminal domains $\mathrm{G} 1$ and $\mathrm{G} 2$ and the $C$-terminal domain G3. The chondroitin sulphate domain of chicken aggrecan is smaller than that of rat and human aggrecans and has very distinctive repeat sequences. It has two separate sections, one comprising 12 consecutive Ser-Gly-Glu repeats of 20 amino acids each, adjacent to the other which has 23 discontinuous Ser-GlyGlu repeats of 10 amino acids each; this latter region, $N$-terminal to the former one, appears to be unique to chicken aggrecan. The two regions contain a total of 94 potential chondroitin sulphate attachment sites. Genomic comparison shows that, although chicken exons 11-14 are identical in size to the rat and human exons, chicken exon 10 is the smallest of the three species. This is also reflected in the size of its chondroitin sulphate coding region and in the total number of Ser-Gly pairs. The putative keratan sulphate domain shows $31-45 \%$ identity with the other species and lacks the repetitive sequences seen in the others. In summary, while the linear arrangement of specific domains of chicken aggrecan is identical to that in the aggrecans of other species, and while there is considerable identity of three separate domains, chicken aggrecan demonstrates unique features, notably in its chondroitin sulphate domain and its keratan sulphate domain. Thus different variants of chondroitin sulphate and keratan sulphate domains may have evolved separately to fulfil specific biochemical and physiological functions.
\end{abstract}

\section{INTRODUCTION}

Cartilage contains an extracellular matrix which consists of a highly concentrated solution of proteoglycans embedded in a meshwork of type II collagen fibres [1,2]. The major proteoglycan of cartilage is a large aggregating molecule, aggrecan, with extensive substitutions of chondroitin sulphate and keratan sulphate chains, as well as $\mathrm{N}$ - and O-linked oligosaccharides [1,3-5]. These substituents comprise about $80-90 \%$ of the molecular mass and thereby dominate the properties of the proteoglycan [1]. The ability of this proteoglycan to specifically form a strong non-covalent bond with hyaluronic acid has been shown to be mediated by its $N$-terminal globular domain, in conjunction with link proteins $[6,7]$.

Biochemical and molecular approaches have been used to study the core protein of aggrecan from the cartilage of different animal species. The cDNA sequences encoding the complete protein sequences of Swarm rat chondrosarcoma aggrecan [8] and human chondrocyte aggrecan [9] show about $75 \%$ amino acid identity, with individual domains ranging from $60 \%$ to $100 \%$ conservation. Partial amino acid sequences of aggrecans from bovine cartilage $[10,11]$ and chicken cartilage [12-14] and the complete cDNA sequence of the human fibroblast proteoglycan, versican [15], have also been reported. Interestingly, unlike human cartilage aggrecan, human fibroblast versican contains two epidermal growth factor (EGF)-like repeats $N$ terminal to the lectin-binding domain and has only one link- protein-like globular domain. Isolation of a porcine aggrecan from laryngeal cartilage [16] has shown that, although the two $N$ terminal globular domains $\mathrm{G} 1$ and $\mathrm{G} 2$ are very similar in sequence, they do not share hyaluronic acid binding activity or immunogenic properties.

In the present study we describe the molecular cloning and nucleotide sequence encoding the entire core protein of chicken sternal cartilage chondroitin sulphate proteoglycan. The deduced amino acid sequences reveal both similarities with and differences from aggrecans of other animal species. While the tandem arrangement of various domains is identical in these aggrecans, chicken aggrecan has unique repetitive sequences in the chondroitin sulphate domain, whereas it lacks any repeat sequences in the keratan sulphate domain, in contrast to the bovine and human homologues. The composite data indicate that different variants of the chondroitin sulphate and keratan sulphate domains may have evolved to fulfil similar biochemical functions.

\section{EXPERIMENTAL}

Materials

Chicken embryos (14-15 days old) were obtained from the University of Connecticut Agricultural Station, Storrs, CT, U.S.A. Reagents for biochemical and molecular cloning experiments were of the highest quality available from commercial vendors.

Abbreviations used: G1, globular domain 1; G2, globular domain 2; G3, globular domain 3; EGF, epidermal growth factor; CRP, complement regulatory protein.

* To whom correspondence should be addressed.

The nucleotide sequence data reported in this paper have been submitted to the GenBank/EMBL Data Bank with accession number M88101. 


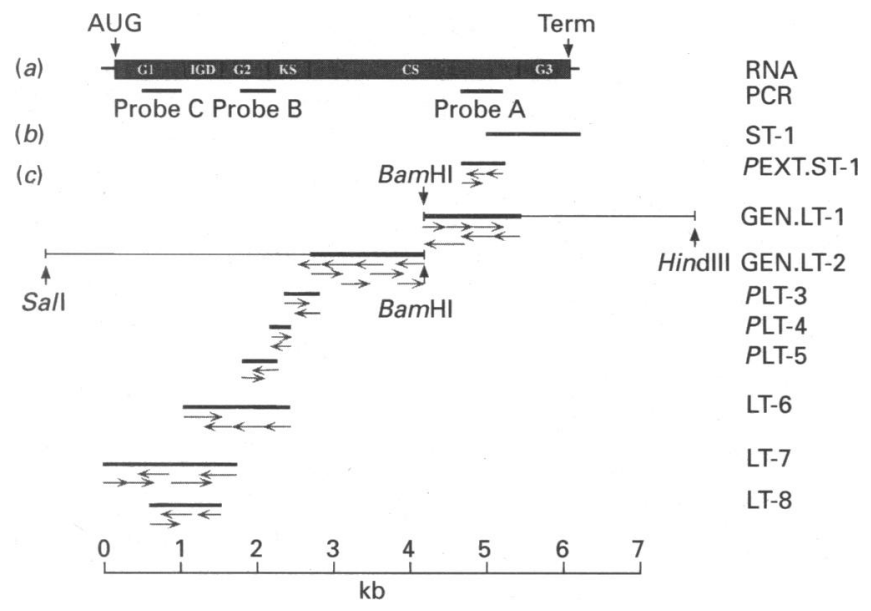

Fig. 1. Cloning and sequencing strategy of the cDNA clones for chicken aggrecan

Relative sizes and locations of the clones are indicated. (a) Composite RNA showing the translational initiation codon (AUG), the structural domains of the protein as a shaded box and the termination codon (Term). The horizontal lines at each end are the $5^{\prime}$ and $3^{\prime}$ untranslated regions. (b) ST-1 clone encoding the $C$-terminal G3 domain was reported earlier [12]. (c) cDNA and genomic clones encompassing the remainder of the coding region of chicken aggrecan. PEXT.ST-1, PLT-3, PLT-4 and PLT-5 are PCR products prepared by the RACE procedure [18] and subcloned into pUC9. GEN.LT-1 and GEN.LT-2 are genomic clones isolated using PEXT.ST-1 (probe A). LT-6, LT-7 and LT-8 are cDNA clones isolated using probes B and C. Solid boxes indicate coding regions. Arrows under the boxes indicate sequencing reactions.

\section{PCR amplification}

Total RNA was prepared from 15-day-old chicken sternal cartilage tissue following the method of Han et al. [17]. Poly(A) ${ }^{+}$ RNA was isolated by passing total RNA through an oligo(dT) column. It was reverse transcribed using Moloney murine leukaemia virus reverse transcriptase and primed with a 17 -mer oligonucleotide unless otherwise mentioned. The single-stranded cDNA was then amplified using the PCR method according to Frohman et al. [18] for $5^{\prime}$ end amplification. Probes A (PEXT.ST-1), B (PLT-5), PLT-3 and PLT-4 were obtained by this method (see Fig. 1).

In order to make probe $\mathrm{C}$ (see Fig. 1) the cDNA library in a $\lambda$ gt10 vector was used as a template for PCR amplification, in which case the $\lambda$ gt10 forward or reverse primer plus a 17 -mer oligonucleotide primer specific for the chicken core protein sequences were used for amplification.

\section{Chicken genomic library screening}

A partial $M b o$ I-digested genomic library in $\lambda$ EMBL3 vector was obtained from Clontech (La Jolla, CA, U.S.A.) and was screened using probe $\mathrm{A}$, which had been isotopically labelled by the random primer extension method [19]. Four positive clones were obtained out of the $6 \times 10^{5}$ plaques which were screened. These clones were purified and were found to have inserts ranging in size from 13 to $16 \mathrm{~kb}$. The largest clone was mapped by restriction enzyme digestions. A $3.5 \mathrm{~kb} \mathrm{BamHI/HindIII} \mathrm{frag-}$ ment (GEN.LT-1; Fig. 1) and a $5 \mathrm{~kb}$ BamHI/Sall fragment
(GEN.LT-2; Fig. 1) were subcloned into Bluescript SK II + by an in-gel ligation method [20].

\section{Chicken cDNA library screening}

Total RNA was prepared from 14-15-day-old chicken sternal cartilage [17]. cDNA synthesis was done by Clontech using the method of Gubler \& Hoffman [21], following poly(A)+ selection as noted above. The cDNA library was prepared as follows. Three separate reactions, primed with an oligo(dT) primer, a random hexanucleotide primer or a specific 17-mer oligonucleotide primer (based on the chicken core protein sequence), were carried out for the first-strand synthesis. The products were pooled for second-strand synthesis to provide equal presentation of the three cDNAs in the library. The cDNA mixture was then cloned into the EcoRI site of $\lambda$ gt10 by conventional methods [22]. When this library was screened using radiolabelled probe $B$ (Fig. 1), approx. one out of $2 \times 10^{3}$ plaques was positive. Five of these clones were chosen for further purification and their EcoRI fragments, ranging in size from $1.4 \mathrm{~kb}$ to $2.2 \mathrm{~kb}$, were subcloned into Bluescript SK II + . One of these five clones, LT-6 (Fig. 1), was sequenced.

The $\lambda$ gt 10 cDNA library was also screened using radiolabelled probe $\mathrm{C}$ to obtain clones which extended further in the $5^{\prime}$ direction. One out of $4 \times 10^{4}$ plaques was positive. Two clones (LT-7 and LT-8; Fig. 1) were chosen for further purification and sequencing.

\section{Nucleic acid sequencing}

The dideoxy chain termination method of Sanger et al. [23] was used to sequence double-stranded templates. All regions of the cDNA were sequenced at least once on both strands. Sequencing of the clones was done using universal primers. As sequence determination progressed, new primers, usually of 15-17-mer, were synthesized based on the ongoing analyses on an Applied Biosystems DNA Synthesizer using phosphoramidite chemistry.

\section{Computer analyses}

Analysis of the deduced amino acid sequence, including database searches and alignments of similar sequences, was performed using the University of Wisconsin Genetics Computer Group programs [24].

\section{RESULTS AND DISCUSSION}

A combination of PCR amplification and genomic and cDNA sequencing resulted in the isolation of nine clones, which together with the initial clone ST-1 [12] accounted for the entire coding sequence of chicken aggrecan core protein (Fig. 1). The composite sequence is 6117 bp long, encodes 1951 amino acids and includes 172 nucleotides of $5^{\prime}$ and 92 nucleotides of $3^{\prime}$ untranslated sequence (Fig. 2). The nucleotide sequence immediately upstream of the initiating codon is in agreement with the consensus sequence for translational initiation in eukaryotes $[25,26]$. Chicken aggrecan core protein is rich in serine, threonine and glutamic acid residues and has a calculated pI of 4.09. There is a total of 29 cysteines, most of which are located in the $C$ - and $N$-terminal globular domains. There is one cysteine in the signal peptide sequence which is not conserved in the other species of aggrecan. There are 94 Ser-Gly pairs and 9 Asn-Xaa-Ser/Thr

\section{Fig. 2. Nucleotide and deduced amino acid sequences of chicken aggrecan}

The boxed sequence represents ST-1. The putative signal peptide cleavage site is indicated by an arrow between residues 20 and 21 . Positions of nucleotide difference between clones LT-6 and LT-8 are at nucleotides 1258 and 1259; in the clone LT-8 they are G and G in place of C and C in clone LT-6. These differences were confirmed by sequencing both strands of overlapping clones in this region. 


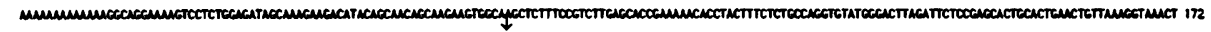

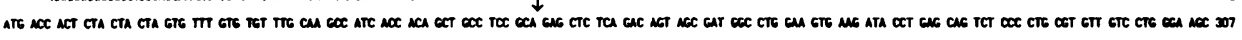

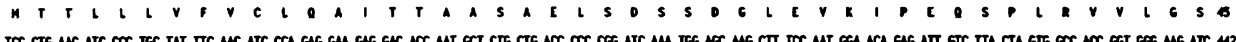

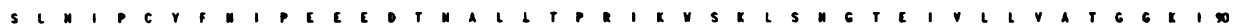

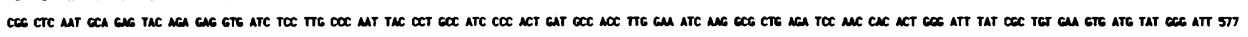

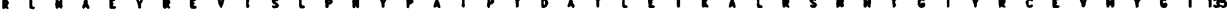

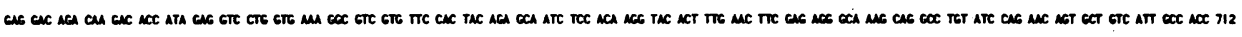

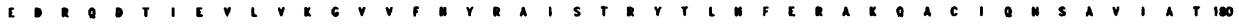

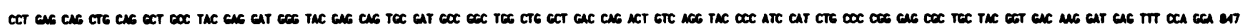

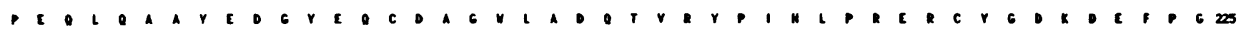

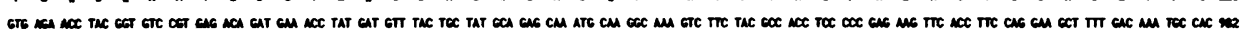

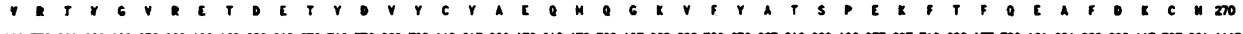

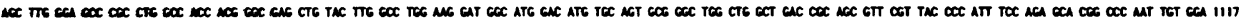

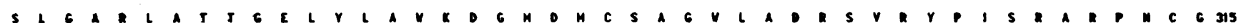

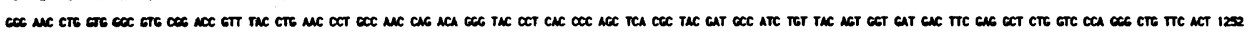

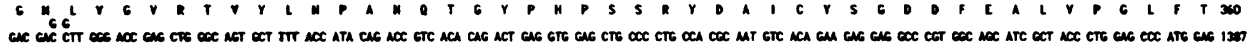

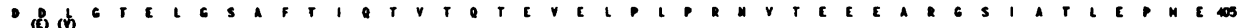

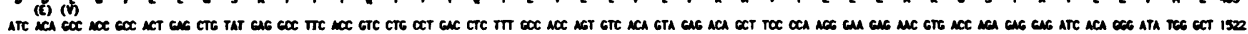

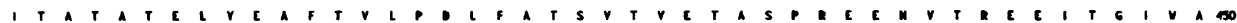

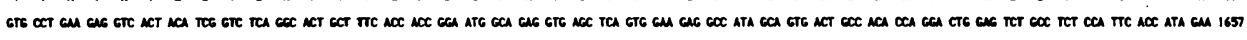

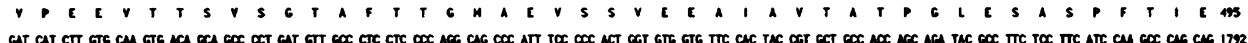

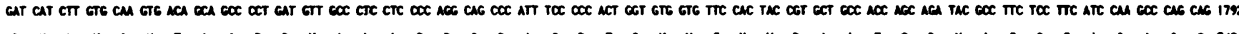

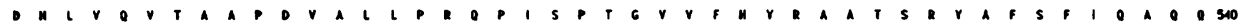

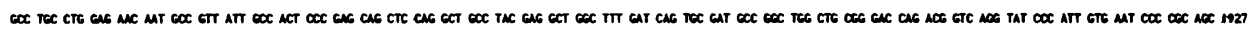

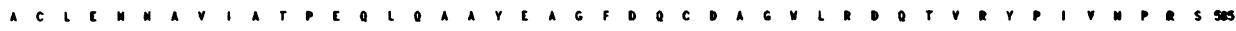

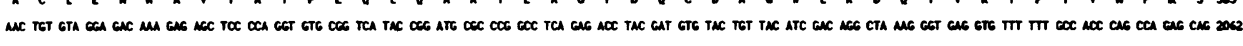

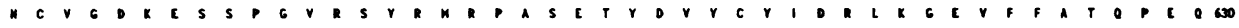

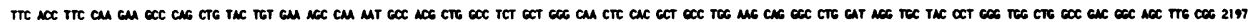

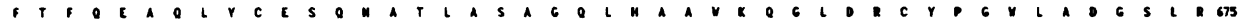

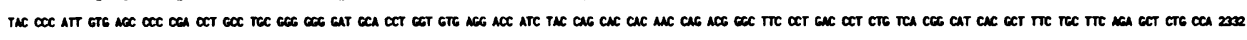

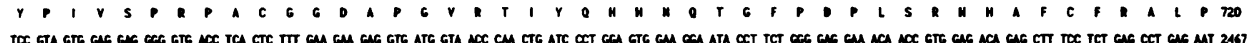

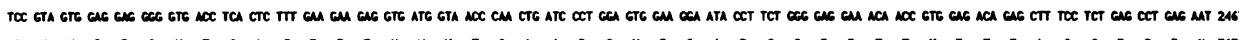

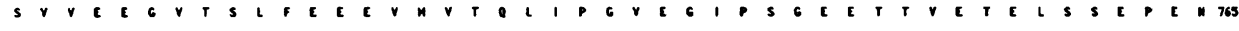

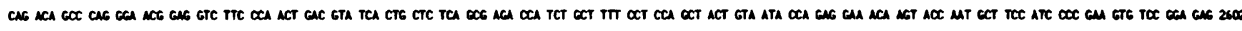

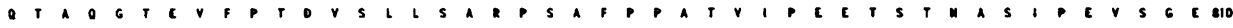

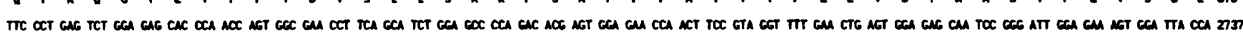

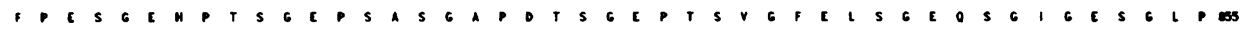

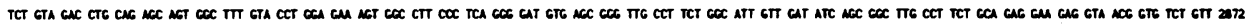
S V D L O S S G F V P G E S G L P S G D V S G L P S G I V D I S G L P S A E E E V T V S V 900

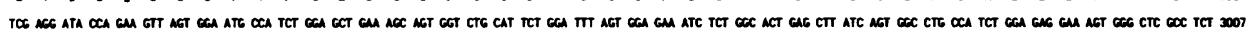

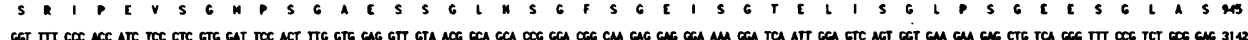

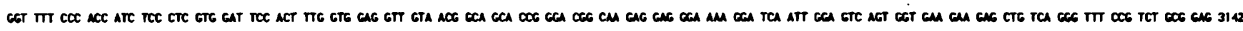

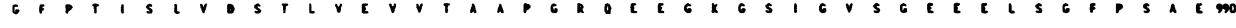

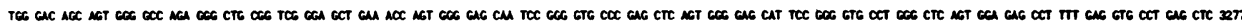
V D S S G A R G L R S G A C T S G E O S G V P E I S G E N S G V P G I S G E P F E V P I I 1030

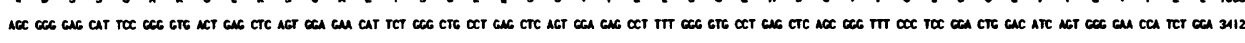

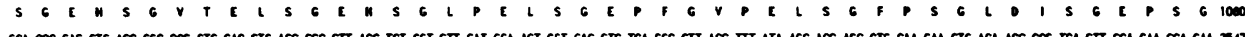

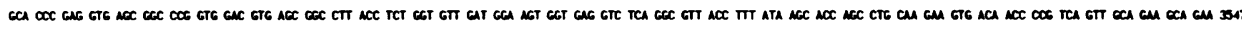
A P I S G P V V V S G I T S G V D G S G I V S G V T F I S T S L E E V T T P S V A E A C 1120

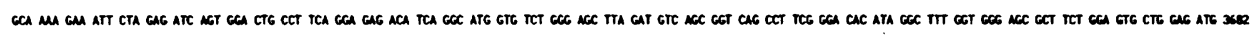

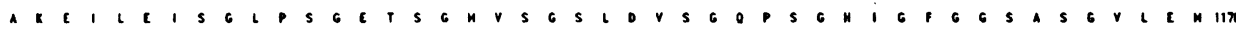

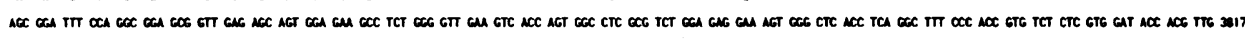
S G : . G G A V E S S G I A S G V E V T S G L A S G E E S G I T S G F P I V S L V D T T I 1215

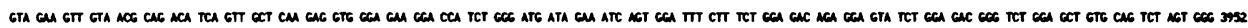

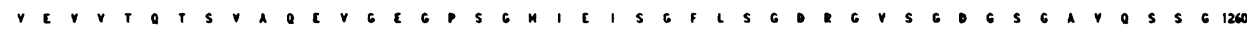

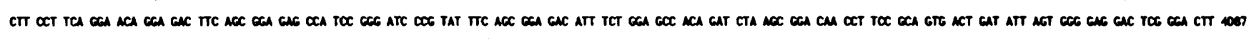

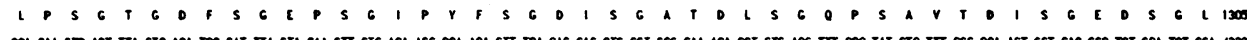

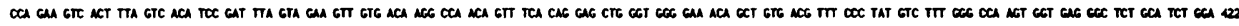

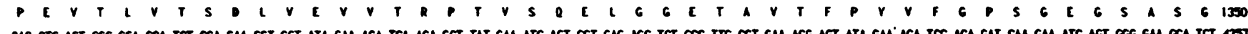

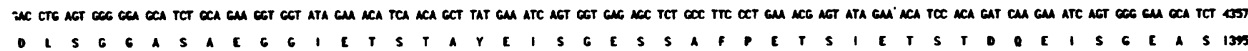

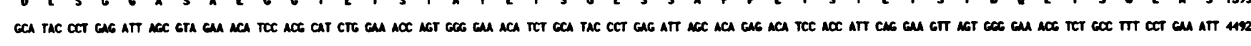

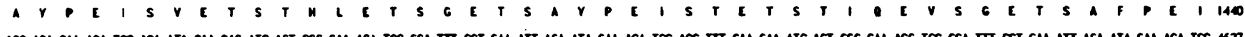

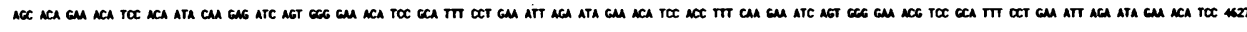
S T E T S T I O E I S G I T S A F P I I I E T S T F E I S G E T S A F E I I I T S 140

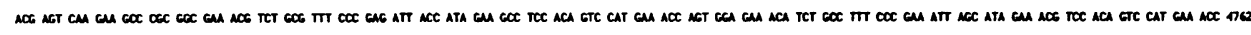
T S O E A R G C T S A F P I T I E A S T V A E T S G E T S A F P I I S I E T S T V F E T 1500

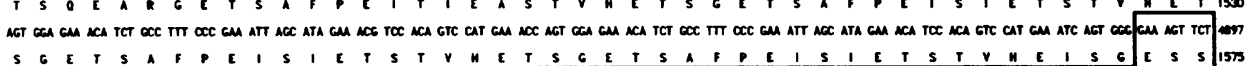

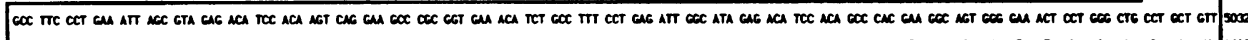

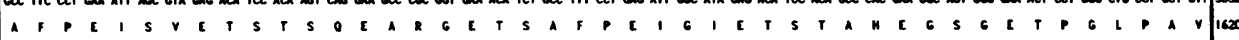

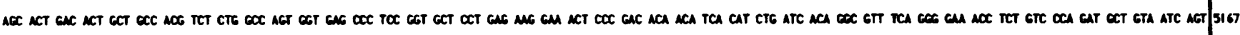

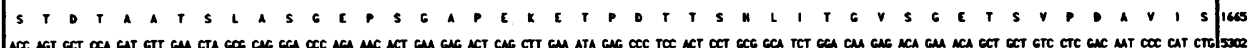

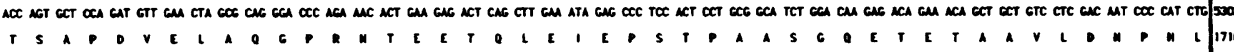

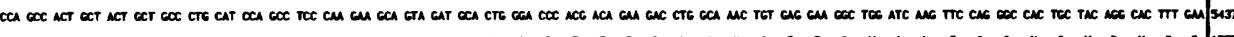

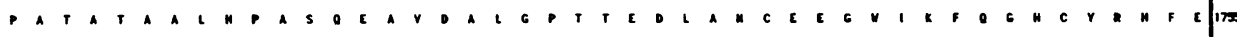

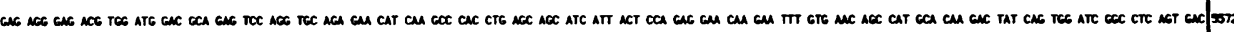

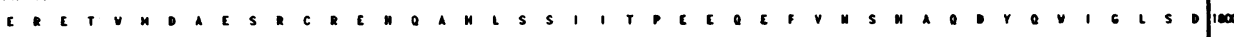

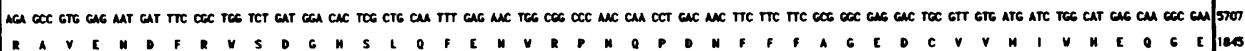

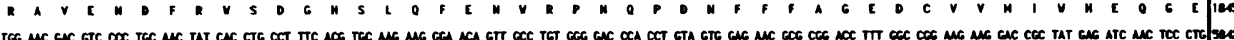

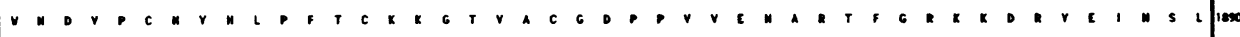

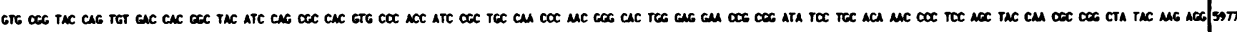

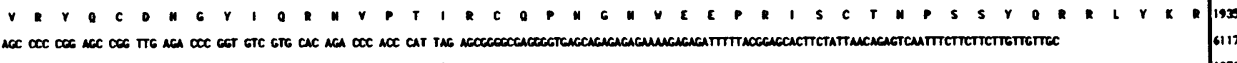

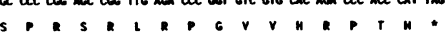


motifs in the deduced sequence. The serine-threonine clusters in the sequence may serve as acceptors for O-linked carbohydrates [27], while the Asn-Xaa-Ser/Thr motifs are potential $N$-glycosylation sites [28]. The signal peptide sequence consists of the $\mathrm{N}$ terminal 20 amino acid residues, with a putative cleavage site between amino acid residues Ala-20 and Glu-21, in accordance with the $(-3,-1)$ rule of von Heijne $[29,30]$.

It is unlikely that the cloned fragments encompass the entire mRNA for the chicken aggrecan. Sai et al. [12] have shown that the mRNA for the core protein of chicken sternal cartilage is about $8.1 \mathrm{~kb}$ by Northern hybridization, whereas the cloned sequences account for only $6.1 \mathrm{~kb}$. It is possible that the $3^{\prime}$ end of the ST-1 clone contains polyadenylation consensus sequences which may partially account for the discrepancy.

Comparison of the deduced amino acid sequence of clone ST1 with that of rat aggrecan showed that it partially encompassed exon 10, which encodes the chondroitin sulphate domain of the proteoglycan core protein. We thus decided to make a probe specific for exon 10 of chicken aggrecan by the RACE method using an mRNA template [18], which could then be used to screen a genomic library. Products of such of PCR amplification almost always appeared as a smear on a $4 \%$ non-denaturing polyacrylamide gel (results not shown). The core-protein-specific product was selected by cloning the PCR products into a pUC 9 vector and screening the resultant library with a nested oligonucleotide probe. One such clone was PEXT.ST-1 (Fig. 1), a $550 \mathrm{bp}$ fragment that was fully sequenced before being used as a probe to screen the chicken genomic library. Southern blots of restriction enzyme digests of the largest clone probed with PEXT.ST-1 showed hybridization with a $3.5 \mathrm{~kb}$ BamHI/HindIII fragment, GEN.LT-1 (Fig. 1). Subcloning and sequencing of this fragment showed an exon sequence of $1.2 \mathrm{~kb}$ which overlapped with the $5^{\prime}$ end of clone ST-1. The deduced amino acid sequence of the exon sequence contained characteristic Ser-Gly repeats, showing it was the chondroitin sulphate domain. In order to complete the exon sequence, the adjacent $5 \mathrm{~kb}$ BamHI/SalI fragment (GEN.LT-2; Fig. 1), which includes exon 10 and a part of its adjacent $5^{\prime}$ intron, was sequenced. In order to precisely determine the $5^{\prime}$ end of exon 10 in the genomic clone, we repeated the PCR amplification using chicken sternal poly $(\mathrm{A})^{+}$ RNA as a template and amplified both the penultimate overlapping sequences of exon 10 and those codon sequences which were $5^{\prime}$ to exon 10 .

Comparison of the partial gene structures of chicken [31] and human [32] aggrecans and the complete structure of the rat gene $[32,33]$ shows that, although exons 11-14 (encoding the $C$ terminal G3 domain) are similar in size, chicken exon 10 is the smallest of the three, being $2.8 \mathrm{~kb}$ in size compared with $3.9 \mathrm{~kb}$ in the human and $3.7 \mathrm{~kb}$ in the rat. In all three species, the entire chondroitin sulphate domain, which constitutes about $50 \%$ of the total protein sequence, is encoded by a single large exon.

There seems to be striking correspondence between the structural domains and corresponding exons of the chondroitin sulphate proteoglycan core proteins, with the exception of the G3 domain, which is considered to be a combination of several motifs. In the case of human aggrecan the G3 domain is made up of a lectin-like motif and an EGF-like motif [34]. There is an additional complement regulatory protein (CRP)-like motif similar to versican [15]. Human aggrecan and versican have molecular isoforms which arise by alternative splicing $[34,35]$. The G3 domain of chicken core protein contains the lectin-like motif and CRP-like motif, but not the EGF-like motif. It has been proposed that the $\mathrm{G} 3$ domain of aggrecans could be composed of two or more segments derived from different genes by exon shuffling $[31,34,35]$.

The $C$-terminal 216 amino acids of chicken aggrecan, residues

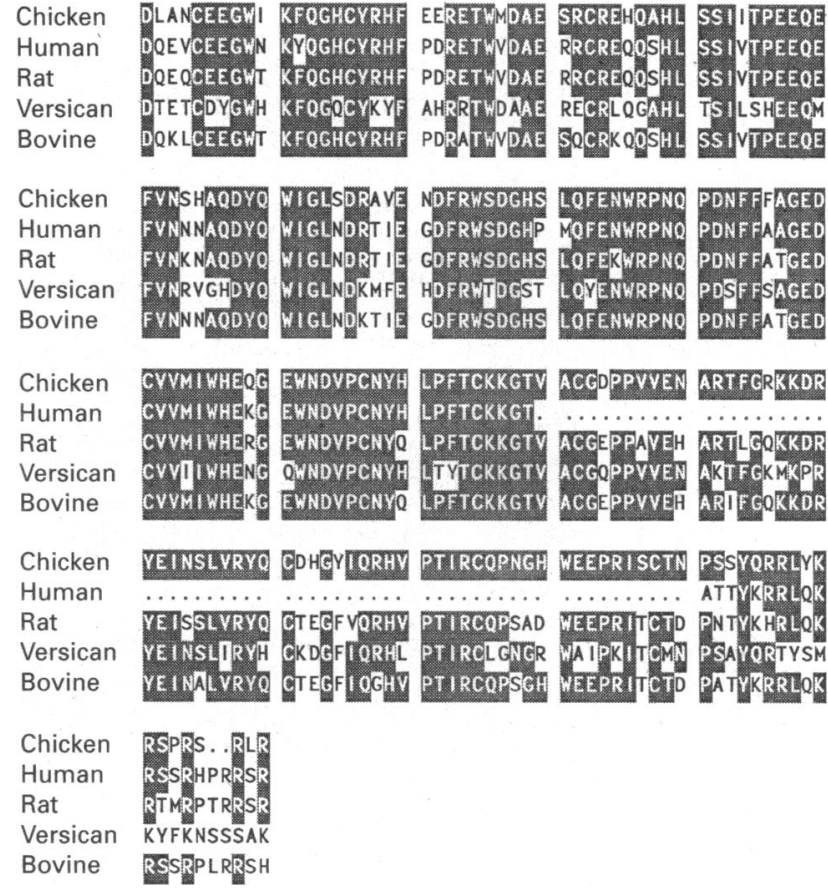

Fig. 3. Comparison, by computer alignment, of the $\mathbf{G 3}$ domains of various aggrecans

The PILEUP program of the Genetics Computer Group Software package was used to compare the G3 domain of chicken with those of the other species. Shaded boxes show identical amino acids. Amino acid residues compared in this Figure are chicken aggrecan 1735-1942, human aggrecan 2163-2311 [9], rat aggrecan 1910-2119 [8], human versican 2178-2387 [15] and bovine aggrecan 505-714 [10].

1735-1951, constitute the globular domain G3. Alignment of this region with the $\mathrm{G} 3$ domains of human, rat and bovine aggrecans and with human versican is shown in Fig. 3. The G3 domain is highly conserved among the different species, showing $70-80 \%$ identity of amino acid sequences. This domain was previously cloned by Sai et al. [12] along with a portion of the chondroitin sulphate domain. It has been suggested by Doege et al. [36] that the lectin-like G3 domain may be involved in binding to carbohydrate residues of cartilage matrix protein or other cartilage matrix components, thereby stabilizing the extracellular matrix. The presence of two different lectin-binding domains, at each end of the aggrecan molecule, potentially enables it to act as a large strut, bridging between other components of cartilage extracellular matrix.

The chondroitin sulphate attachment domain of chicken aggrecan seems to be unique in its size and structure (Fig. 4). Compared with bovine nasal cartilage proteoglycan core protein, the distance between the second globular domain G2 and the third globular domain $\mathrm{G} 3$, constituting the chondroitin sulphate domain for the most part, was found to be shorter in rotaryshadowed images of chicken cartilage proteoglycan core protein [37]. Also, the cDNA encoding the chondroitin sulphate domain of chicken aggrecan fails to recognize rat chondrosarcoma RNA [14], which suggests that there are considerable differences between their sequences.

The chondroitin sulphate domain of chicken core protein is comprised of 903 amino acids (residues 800-1702) and has several interesting features. It can be divided into two subdomains: an $N$-terminal region (CS-1) and a $C$-terminal region (CS-2), as shown in the dot matrix plot in Fig. 4. The CS-2 region (Fig. 4c) shows extensive repetitive sequences of 20 amino acids, 

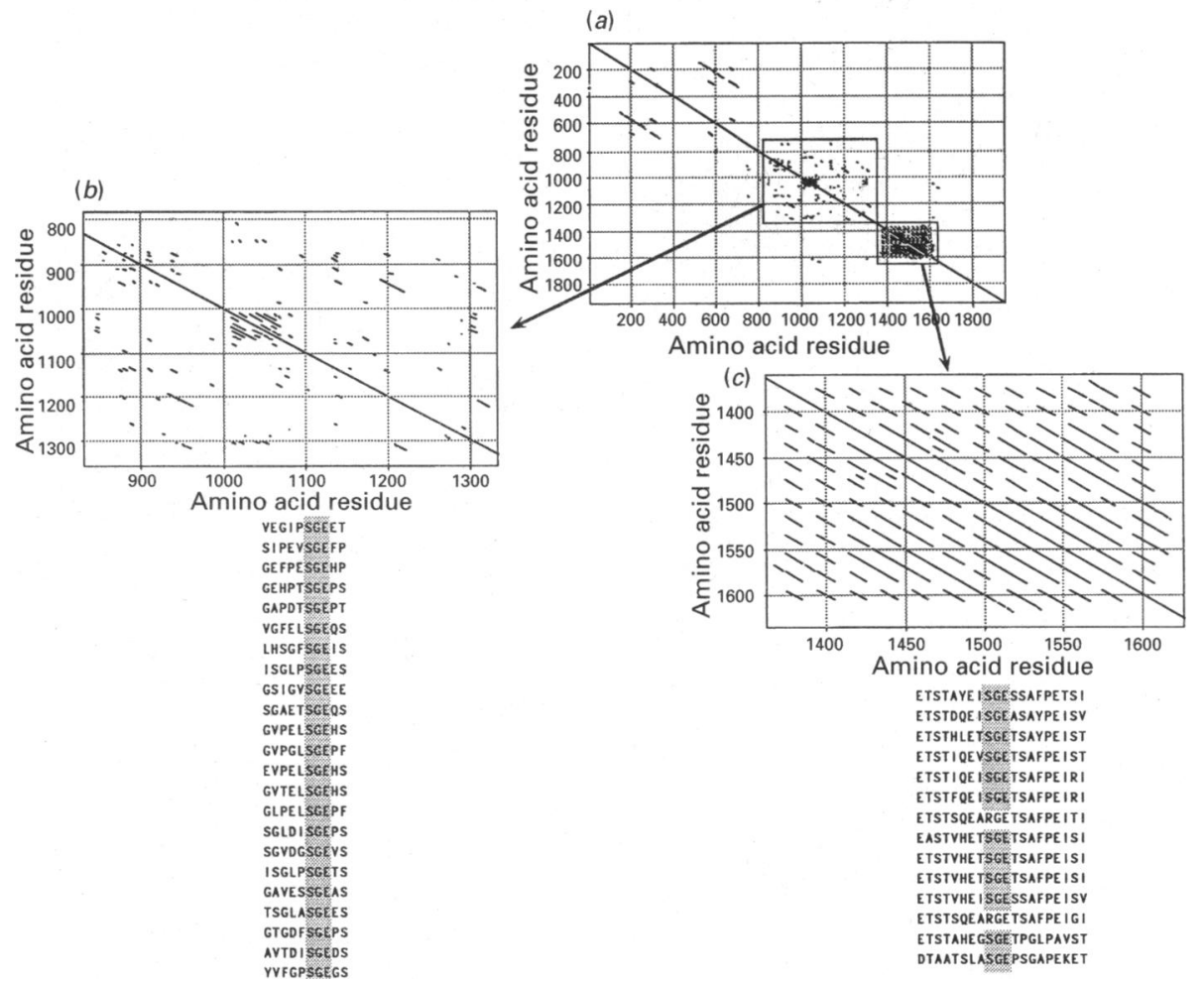

Fig. 4. Dot matrix plots of chicken aggrecan

(a) Plot for the full-length protein. Areas within the inset squares show extensive repeats present in the chondroitin sulphate domain; (b) 10-aminoacid repeats in the CS-1 region with their SGE sequences aligned (residues 744-1347); (c) 20-amino-acid repeat sequences present in the CS-2 region with their SGE sequences aligned (residues 1363-1642). The MACVECTOR program (IBI Biotechnologies) was used for this analysis.

with $16-19$ of the amino acids often being identical. These 20 residues are repeated 12 times with two interruptions. Both of these interruptions could result from single base mutations from A to C (nucleotides 4643 and 4943), resulting in a Ser to Arg change. The potential consensus sequences for $O$-xylosylation by xylosyltransferase [38], Xaa-Ser-Gly and Ser-Gly-Xaa-Gly, are seen within the 20 residue repeats. The adjacent CS- 1 region has 23 discontinuous repeats of 10 amino acids each (Fig. $4 b$ ), with potential xylosyltransferase recognition sequences three to five residues apart. Oldberg et al. [10] and Krueger et al. [13] have speculated that such motifs could generate an amphiphilic $\beta$ strand secondary structure, which might enhance substrate acceptor capability for xylosylation.

Chicken aggrecan has a total of 94 potential chondroitin sulphate attachment sites: 12 are in the 20 -fold repeat CS- 2 region, 77 are in the 10 -fold repeat CS- 1 region, and the remainder are in flanking sequences. Overall, the chondroitin sulphate domain of chicken aggrecan shows only $45 \%$ identity with the

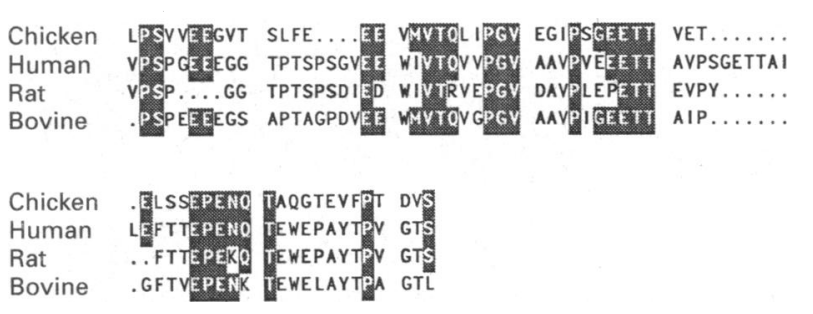

Fig. 5. Comparison, by computer alignment, of the keratan sulphate domains

Amino acid residues compared are chicken aggrecan 719-779, human aggrecan 679-751 [9], rat aggrecan 689-749 [8] and bovine aggrecan 128-191 [11]. Refer to Fig. 3 for other details.

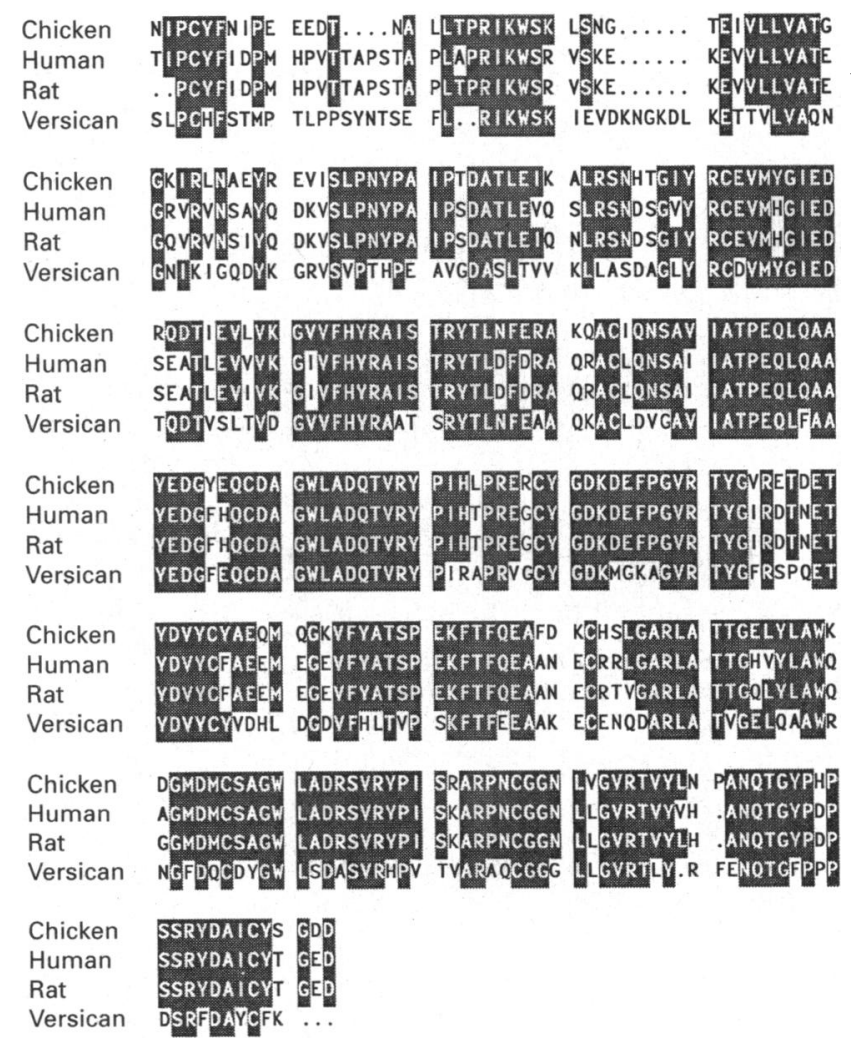

\section{Fig. 6. G1 domain comparisons}

Amino acid residues compared are chicken aggrecan 48-350, human aggrecan 48-353 [9], rat aggrecan 50-353 [8] and human versican 41-347 [15]. Refer to Fig. 3 for other details. 


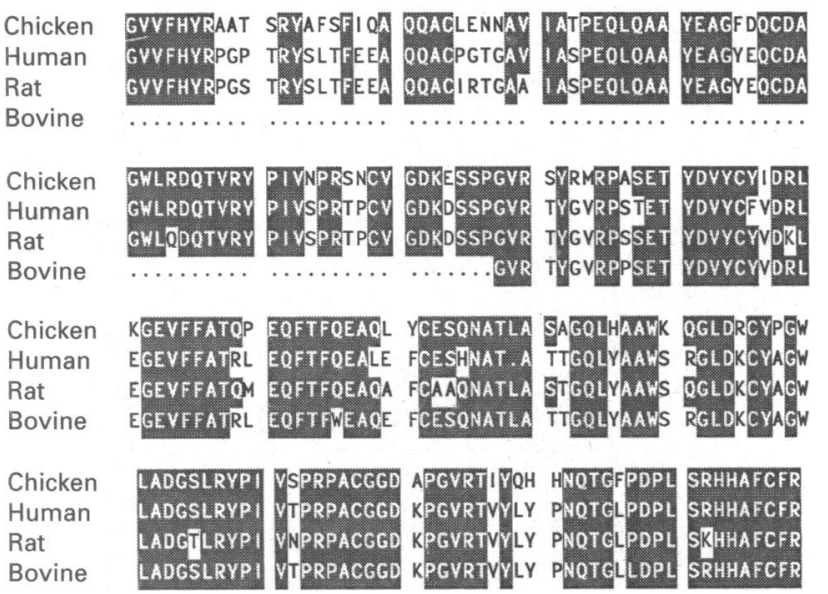

Fig. 7. Computer alignment of G2 domains

Amino acid residues compared are chicken aggrecan 519-717, human aggrecan 477-674 [9], rat aggrecan 486-684 [8] and bovine aggrecan 1-122 [11]. Refer to Fig. 3 for other details.

equivalent rat/human domains and $27 \%$ identity with human versican.

Comparison of the amino acid sequence of the chondroitin sulphate-derived peptide CS-B and the deduced sequence of a corresponding cDNA clone, expressed as an antibody-reactive protein, revealed a single amino acid difference, Asp versus Glu respectively, between them [14]. We found, in the genomic clone GEN.LT-2 (Fig. 1) which overlaps this region, that the corresponding codon third base position is $\mathrm{C}$ (nucleotide 3925; Fig. 2) denoting an Asp residue, as found in the peptide.

The keratan sulphate domain by definition is that part of the core protein that bears the majority of the keratan sulphate chains and very few chondroitin sulphate chains. This domain has not been well characterized to date. In general, it is found to be rich in glutamic acid, glutamine, proline and serine residues, which constitute about $65 \%$ of the amino acid residues in this region [2]. The core proteins of human and bovine aggrecans possess characteristic hexameric repeat sequences which are rich in glutamic acid, proline and serine residues $[9,11]$.

Cyanogen bromide peptide mapping by Haynesworth et al. [39] has shown that chicken cartilage proteoglycan core protein contains a small fragment $(47 \mathrm{kDa})$ that is predominantly rich in keratan sulphate chains. The putative keratan sulphate domain of chicken aggrecan cDNA was assigned based on comparison with rat, bovine and human aggrecans, assuming that the $C$ terminal end of this domain is continuous with the $\mathrm{N}$-terminal end of the chondroitin sulphate domain. As shown, the keratan sulphate domain of chicken aggrecan lacks any hexameric repeat sequence (Fig. 5). It shows a range of $31-45 \%$ amino acid identity with the keratan sulphate domains of rat, human and bovine aggrecans. Also, there is a potential $N$-glycosylation site in this domain which is conserved in the human and bovine aggrecans.

The hyaluronic acid binding domain of proteoglycan core protein consists of two tandem globular domains, G1 and G2 [8], separated by an interglobular region, IGD. The two globular domains have a high degree of similarity to each other and to link protein. The G1 domain of chicken aggrecan has 10 conserved cysteine residues and two $\mathrm{N}$-glycosylation sites, only one of which is conserved in human versican. Comparison of the G1 domain of chicken aggrecan with those of human and rat aggrecans and versican is shown in Fig. 6. Data for the bovine G1 domain are not available for comparison but, considering

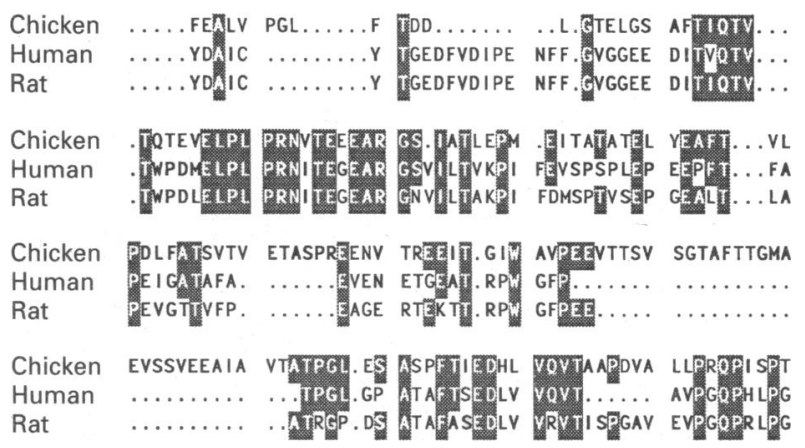

Fig. 8. Comparison of the interglobular domain sequences of different species

Chicken aggrecan residues 351-518 are compared with human aggrecan residues 344-477 [9] and rat aggrecan residues 344-485 [8]. Refer to Fig. 3 for other details.

that the G2 domain of bovine aggrecan shows $78 \%$ identity with the chicken $\mathrm{G} 2$ domain and that the $\mathrm{G} 1$ and $\mathrm{G} 2$ domains of aggrecans are very similar, it is likely that the G1 domain of bovine aggrecan would be very similar to the G1 domain of chicken aggrecan.

Comparison of the $\mathrm{G} 2$ domains of chicken, rat, human and bovine aggrecans is shown in Fig. 7. Once again, 75-78\% of the amino acid residues in this domain are identical among the different species. This domain has eight conserved cysteines and two potential $N$-glycosylation sites. Because the two domains, $\mathrm{G} 1$ and $\mathrm{G} 2$, are more similar to each other than to link protein, it has been proposed that the proteoglycan gene evolved by duplication of the sequences following the divergence of the ancestral genes [40]. The deduced amino acid sequence of the interglobular domain of chicken was compared with that of rat and human (Fig. 8). It is interesting to see that this domain, which lies between two very similar domains, matches poorly between aggrecans, with only $30-40 \%$ of the residues being identical. It has been shown by electron microscopy that the interglobular domain has an extended appearance [41], and is rich in acidic residues and proline. The function of this domain is not very clear and it may simply serve to unite the G1 and G2 domains.

Link protein is structurally similar to the $N$-terminal $\mathrm{G} 1$ and G2 domains of aggrecan and also binds to hyaluronic acid [42]. Link protein primary structure in different species also shows a high degree of conservation. Comparison of chicken link protein sequence [43] with chicken aggrecan G1 and G2 domains shows $40 \%$ identity (results not shown), in contrast to the $70 \%$ identity between G1 and G2 themselves. Similar results have been reported for rat link protein and rat aggrecan [40]. Thus, while there are strong similarities between link protein and aggrecan domains, they probably diverged earlier than the subsequent duplication of the initial aggrecan exon, as noted above.

Dendrograms were prepared to show the clustering relationship of the order of pairwise alignments of various domains of aggrecan among different species (Fig. 9). The clustering is arranged by the computer program so that very similar sequences are aligned to each other before they are aligned to more distantly related sequences. The dendrograms presented in Fig. 9 are not intended to represent phylogenetic reconstructions, although the lengths of the horizontal lines are proportional to the similarity between the sequences. For example, the keratan sulphate domains of human and rat aggrecans are more similar to each other compared with bovine aggrecan, whereas the G3 domains of rat and bovine aggrecans are more similar compared with the human. Another interesting finding is that the different 

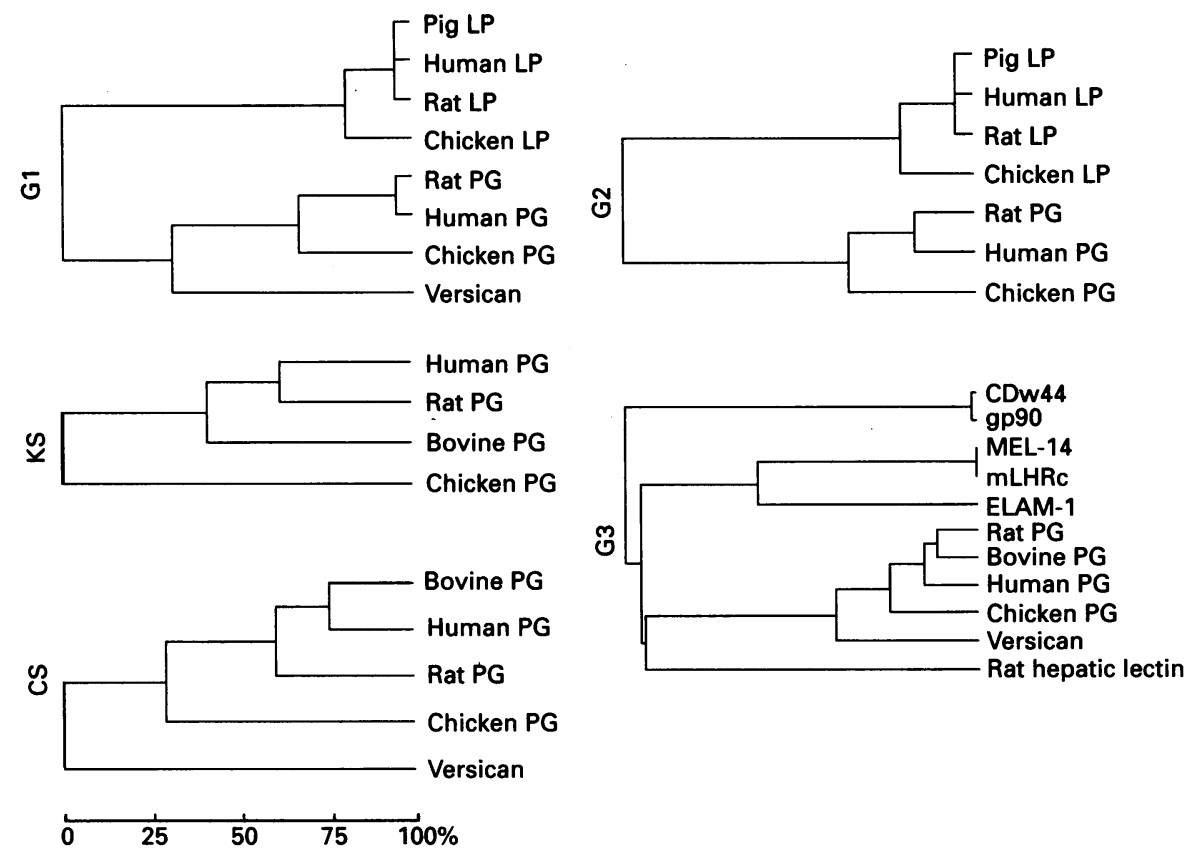

Fig. 9. Dendrograms relating the sequences of the different domains of aggrecans, link proteins and various lectins

The dendrograms were constructed by comparison of the amino acid sequences of G1, G2, G3, keratan sulphate (KS) and chondroitin sulphate (CS) domains of aggrecans and versican among themselves, to link proteins, to a hepatic lectin and to lymphocyte homing receptors. The scale shown at the bottom represents the relative sequence similarity applicable to the horizontal lines of all of the dendrograms. Distances along the horizontal axis are proportional to the difference between sequences; distance along the vertical axis has no significance. References for the sequences compared are proteoglycans (PG): aggrecan core protein from rat [8], human [9], bovine [10,11] and versican [15]. Link proteins (LP) are from rat $[42,44]$, chicken [43] pig [45] and human [46]. Hepatic lectin is from rat [47]. Endothelial cell adhesion molecule (ELAM-1) [48] and lymphocyte homing receptors are from mouse, MEL-14 [49] and mLHRc [50], and human CDw44 [51] and gp90 [52]. The PILEUP program of the Genetics Computer Group software package was used to generate the dendrograms.

domains of chicken aggrecan are distantly related to the corresponding human, rat or bovine domains. The $G 1$ and G2 domains of link proteins and aggrecans cluster separately and show the same inter-species relatedness in each cluster. In the case of the G3 domain, the various lectin-like domains fall into separate clusters which are distantly related to each other. In this instance, the clusters of proteoglycans show considerable relatedness to each other. This degree of structural conservation suggests that the G3 domain has an important role in aggrecan structure, a role whose physiological relevance remains to be determined.

In summary, we report the full-length cDNA sequence which codes for chicken aggrecan. The comparison of the sequences of the different domains of the chicken molecule with those of the rat and human, for which complete coding sequences are also available, provides some insight into the evolution of this large multi-domain molecule.

This work was supported by grant no. 5P01-HD22610 from NIH.

\section{REFERENCES}

1. Hassell, J. R., Kimura, J. H. \& Hascall, V. C. (1986) Annu. Rev. Biochem. 55, 539-567

2. Heinegård, D. \& Paulson, M. (1984) in Extracellular Matrix Biochemistry (Piez, K. A. \& Reddi, A. H., eds.), pp. 277-328, Elsevier, New York

3. Heinegård, D. \& Axelsson, I. (1977) J. Biol. Chem. 252, 1971-1979

4. Lohmander, L. S., Deluca, S., Nilsson, B., Hascall, V. C., Caputo, C. B., Kimura, K. H. \& Heinegård, D. (1980) J. Biol. Chem. 255, 6084-6091

5. Nilsson, B., De Luca, S., Lohmander, L. S. \& Hascall, V. C. (1982) J. Biol. Chem. 257, 10920-10927
6. Muir, I. H. M. (1980) in The Joints and Synovial Fluid (Sokolof, L., ed.), vol. 2, pp. 27-94, Academic Press, New York

7. Hascall, V. C. (1977) J. Supramol. Struct. 7, 101-120

8. Doege, K., Sasaki, M., Horigan, E., Hassell, J. R. \& Yamada, Y. (1987) J. Biol. Chem. 262, 17757-17767

9. Doege, K., Sasaki, M., Kimura, T. \& Yamada, Y. (1991) J. Biol. Chem. 266, 894-902

10. Oldberg, A.,, Antonsson, P. \& Heinegård, D. (1987) Biochem. J. 243, 255-259

11. Antonsson, P., Heinegård, D. \& Oldberg, Å. (1989) J. Biol. Chem. 264, 16170-16173

12. Sai, S., Tanaka, T., Kosher, R. A. \& Tanzer, M. L. (1986) Proc. Natl. Acad. Sci. U.S.A. 83, 5081-5085

13. Krueger, R. C., Fields, T. A., Hildreth, J. \& Schwartz, N. B. (1990) J. Biol. Chem. 265, 12075-12087

14. Krueger, R. C., Fields, T. A., Mensch, J. R. \& Schwartz, N. B. (1990) J. Biol. Chem. 265, 12088-12097

15. Zimmerman, D. R. \& Ruoslahti, E. (1989) EMBO J. 8, 2975-2981

16. Fosang, A. J. \& Hardingham, T. E. (1989) Biochem. J. 261, 801-809

17. Han, J. H., Stratowa, C. \& Rutter, W. J. (1987) Biochemistry 26, $1617-1625$

18. Frohman, M. A., Dush, M. K. \& Martin, G. R. (1988) Proc. Natl. Acad. Sci. U.S.A. 85, 8998-9002

19. Feinberg, A. P. \& Vogelstein, B. (1984) Anal. Biochem. 137, 266-267

20. Sambrook, J., Fritsch, E. F. \& Maniatis, T. (1989) Molecular Cloning: A Laboratory Manual, 2nd edn. vol. 2, pp. 1.72-1.73, Cold Spring Harbor Laboratory Press, Cold Spring Harbor

21. Gubler, U. \& Hoffman, B. J. (1983) Gene 25, 263-269

22. Huynth, T. V., Young, R. A. \& Davis, R. W. (1985) in DNA Cloning: A Practical Approach (Glover, D. M., ed.), vol. 1, pp. 49-78, IRL Press, Oxford

23. Sanger, F., Nicklen, S. \& Coulson, A. R. (1977) Proc. Natl. Acad. Sci. U.S.A. 74, 5463-5467

24. Devereux, J., Haeberli, P. \& Smithies, O. (1984) Nucleic Acids Res. 12, 387-395

25. Kozak, M. (1987) Nucleic Acids Res. 15, 8125-8132

26. Kozak, M. (1991) J. Cell Biol. 115, 887-903 
27. Wilson, I. B. H., Gavel, Y. \& von Heijne, G. (1991) Biochem. J. 275 , 529-534

28. Marshall, R. D. (1974) Biochem. Soc. Symp. 40, 17-26

29. von Heijne, G. (1983) Eur. J. Biochem. 133, 17-21

30. von Heijne, G. (1986) Nucleic Acids Res. 14, 4683-4690

31. Tanaka, T., Har- El, R. \& Tanzer, M. L. (1988) J. Biol. Chem. 263, 15831-15835

32. Doege, K., Sasaki, M. \& Yamada, Y. (1990) Biochem. Soc. Trans. 18, 200-202

33. Doege, K. \& Yamada, Y. (1988) Collagen Relat. Res. 8, 486

34. Baldwin, C. T., Reginato, A. M. \& Prockop, D. J. (1989) J. Biol. Chem. 264, 15747-15750

35. Perin, J., Bonnet, F., Jollès, J. \& Jollès, P. (1984) FEBS Lett. 176, $37-42$

36. Doege, K., Fernandez, P., Hassel, J. R., Sasaki, M. \& Yamada, Y. (1986) J. Biol. Chem. 261, 8108-8111

37. Dennis, J. E., Carrino, D. A., Schwartz, N. B. \& Caplan, A. I. (1990) J. Biol. Chem. 265, 12098-12103

38. Bourdon, M. A., Krusius, T., Campbell, S., Schwartz, N. B. \& Ruoslahti, E. (1987) Proc. Natl. Acad. Sci. U.S.A. 84, 3194-3198

39. Haynesworth, S. E., Carrino, D. A. \& Caplan, A. I. (1987) J. Biol. Chem. 262, 10574-10581

40. Doege, K., Rhodes, C., Sasaki, M., Hassell, J. R. \& Yamada, Y. (1990) in Collagen Genes: Structure, Regulation \& Abnormalities (Boyd, C. D., Byers, P. \& Sandell, L., eds.) pp. 137-155, Academic Press, Orlando, FL
41. Paulsson, M., Morgelin, M., Wiedemann, H., Beardmore-Gray, M., Dunham, D., Hardingham, T., Heinegård, D., Timpl, R. \& Engel, J. (1987) Biochem. J. 245, 763-772

42. Neame, P. J., Christner, J. E. \& Baker, J. R. (1986) J. Biol. Chem. 261, 3519-3535

43. Deak, F., Kiss, I., Sparks, K. J., Argraves, W. S., Hampikian, G. \& Goetinck, P. F. (1986) Proc. Natl. Acad. Sci. U.S.A. 83, 3766-3770

44. Doege, K., Hassel, J. R., Caterson, B. \& Yamada, Y. (1986) Proc. Natl. Acad. Sci. U.S.A. 83, 3761-3765

45. Perkins, S. J., Nealis, A. S., Dudhia, J. \& Hardingham, T. E. (1989) Genbank Accession no. Y00165

46. Dudhia, J. \& Hardingham, T. E. (1990) Nucleic Acids Res. 18, 1292

47. Halberg, D. F., Wager, R. E., Farrell, D. C., Hildreth, J., Quesenberry, M. S., Loeb, J. A., Holland, E. C. \& Drickamer, K. (1987) J. Biol. Chem. 262, 9828-9838

48. Bevilacqua, M. P., Stengelin, S., Gimbrone, M. A. \& Seed, B. (1989) Science 243, 1160-1165

49. Lasky, L. A., Singer, M. S., Yednock, T. A., Dowbenko, D., Fennie, C., Rodriguez, H., Nguyen, T., Stachel, S. \& Rosen, S. D. (1989) Cell 56, 1045-1055

50. Siegelman, M. H., van de Rijn, M. \& Weissman, I. L. (1989) Science 243, 1057-1062

51. Stamenkovic, I., Amiot, M., Pesando, J. M. \& Seed, B. (1989) Cell 56, 1057-1062

52. Goldstein, L. A., Zhou, D. F. H., Picker, L. J., Minty, C. N., Bargatze, R. F., Ding, J. F. \& Butcher, E. C. (1989) Cell 56, 1063-1072

Received 29 April 1992/22 June 1992; accepted 29 June 1992 